\title{
LA EDICIÓN NACIONAL DE LA OBRA DE BENEDETTO CROCE: ESTÉTICA COMO CIENCIA DE LA EXPRESIÓN Y LINGÜIISTICA GENERAL
}

\author{
DAVIDE MOMBELLI
}

Universidad de Alicante

BenedetTo CRoce, Estetica come scienza dell'espressione e linguistica generale, ed. crítica de Felicita Audisio, Bibliopolis, Nápoles, 2014, 3 vols.

En 2014 vio la luz la Edición Nacional de la Estetica come scienza dell'espressione e linguistica generale en la editorial napolitana Bibliopolis, a cargo de Felicita Audisio, quien ya editó la Filosofía de Giambattista Vico (1997). Con el volumen de la Estética finaliza la publicación de los tomos que componen la «Filosofía del espíritu», que empezó en 1996 con la edición de la Logica come scienza del concetto puro. La demora en la publicación de lo que es el primer texto del sistema filosófico croceano se debe a que la «complessa edizione» de la Estética, según la define la misma Audisio, se planteó sobre las muchas versiones, reescrituras, correcciones que Croce hizo del texto, aumentando así el número de testimonios a colacionar.

Benedetto Croce (1866-1952) es una figura esencial del pensamiento estético occidental. Pese a ello, sobre todo a partir de la segunda posguerra, incluso en Italia los estudios sobre su obra disminuyeron notablemente. Esta especie de ostracismo hacia el gran esteta se debe, principalmente, al mutado clima cultural italiano de las últimas décadas y al triunfo de escuelas críticas de impostación estructuralista, que también en Italia tuvieron gran éxito. Sin embargo,

${ }^{1}$ Grupo de Investigación HUMANISMO-EUROPA. 
hay excepciones muy relevantes que testimonian cómo la «influencia cultural de Benedetto Croce», como la llamaría Gianfranco Contini, siguió estando viva. Es en especial el caso del importante e influyente grupo de investigación Centro Internazionale Studi di Estetica de Palermo, dirigido por Luigi Russo y fundado por éste en 1980. Es significativo que el primer número de la revista vinculada al Centro esté dedicado al filósofo partenopeo (Croce e l'estetica). Decisiva en la continuación de los estudios croceanos es la labor desarrollada desde los años 50 del pasado siglo por los investigadores vinculados al Istituto Italiano per gli Studi Storici, fundado por el mismo Croce en 1946 y ubicado en la que fue su residencia, el palacio Filomarino, en el corazón de Nápoles. Presidente del Istituto es Natalino Irti, y Marta Herling su secretaria académica. En el mismo palacio Filomarino se encuentra también su biblioteca y la Fundación a ella asociada, dirigida por Piero Craveri².

La Edición Nacional de la Estética es una edición crítica realizada con el máximo rigor ecdótico. Está organizada en tres volúmenes, el primero de los cuales recoge la versión de 1904 (2. edición), el segundo el texto de la última versión publicada en vida por Croce (la de 1950) y el tercer tomo presenta, además de un estudio sobre la tradición textual y la fortuna editorial de la obra, todas las variantes y las referencias bibliográficas empleadas por el autor.

Antes de pasar a comentar la edición crítica de la obra y la bibliografía estética sobre y de Croce en España, convendrá precisar el concepto de Edición Nacional, una operación editorial que, creemos, es específica del caso italiano, debido a factores y causas culturales, literarias y políticas.

\section{La Edizione Nazionale: concepto e historia}

Como recuerda Francesco Sicilia en el prefacio a Storia e bibliografia delle edizioni nazionali, el primer objetivo del «Ministero per i Beni e le Attività culturali» es difundir una «consapevolezza generalizzata dell'importanza del nostro patrimonio culturale» $\rangle^{3}$ y garantizar así la tutela, la valorización y difusión del patrimonio literario y de pensamiento constituido por los escritos de una serie de destacados autores italianos. Dentro de este marco, la finalidad de las Ediciones Nacionales es la publicación, por parte de un comité científico, de la opera omnia de un autor, mediante realizaciones de riguroso criterio filológico, fundadas en la colación de todos los textos impresos o manuscritos conocidos.

${ }^{2}$ Para más información sobre el grupo de investigación palermitano, el Istituto Italiano per gli Studi Storici y la Fondazione Biblioteca Benedetto Croce, visítese las respectivas Web: http://wwwl.unipa.it/ estetica/_home.html; https.//www.iiss.it; y http://www.fondazionebenedettocroce.it.

${ }^{3}$ M. Scotti y F. Cristiano, Storia e bibliografia delle edizioni nazionali, Sylvestre Bonnard, Milán, 2002, pág. 9. 
El concepto de Edición Nacional gravita alrededor de otros conceptos colindantes, que se implican y se sobreentienden. Uno de ellos, que aparece también en la definición «oficial» del concepto de Edición Nacional, es el de «patrimonio», término que se relaciona con otros, a saber, «valor cultural» $\mathrm{y}$ «bienes culturales o artísticos». En la especificidad del caso italiano, fundamental es la definición que de «patrimonio cultural» dio la Comisión Franceschini (1964), instituida con el fin de revisar el sistema del ordenamiento jurídico y administrativo de la cultura nacional: «pertenecen al patrimonio cultural de la Nación todos los bienes que se refieren a la historia de la civilización. Quedan sujetos a la ley los bienes de interés arqueológico, histórico, artístico, ambiental y paisajístico, archivístico y bibliográfico, y todos los bienes que constituyan un testimonio material con valor de civilización» ${ }^{4}$. El término «patrimonio» incluye el concepto de «herencia cultural», que puede traducirse también como «tradición», cuya acepción realativa a «acumulación» de hechos (culturales, históricos, sociales) es implícita. Una «acumulación» que no es acrítica y, en el caso de una literatura ligada estrictamente al Estado-Nación, se configura como una construcción deliberadamente dirigida a un objetivo, la definición y sucesiva revalorización de un determinado 'espíritu nacional'. Significativamente, lo que se pudiera considerar el primer ensayo de Edición Nacional, pese a que todavía no se le atribuyó ese nombre, fue patrocinado por Francesco de Sanctis, autor de una de las más importantes historias literarias italianas, entonces ministro de la Instrucción Pública: es en 1879 la edición de la obra latina de Giordano Bruno, una declaración de principios políticos y éticos sobre los que se debía basar la construcción cultural y social del neonato estado italiano. El esfuerzo valorativo de la literatura tanto artística como científica y filosófica nacional es algo que acompañó desde el principio la formación del estado italiano y hasta precedió en muchos años la fundación del aparato político y administrativo. En efecto, data de 1772 la publicación del primer tomo de la Storia della letteratura italiana de Girolamo Tiraboschi ${ }^{5}$, una obra que ve la luz casi un siglo antes de la unificación política de la península (1861), entonces dividida en decenas de estados independientes y sin una lengua vehicular fija (aunque evidentemente existía una lengua literaria común, la fundada sobre el dialecto toscano de los grandes autores del Trecento).

${ }^{4}$ Ley del 26 de abril de 1964, n. 310. En D. Vaiano, La valorizzazione dei beni culturali, Giappichelli, Turín, 2011, pág. 2.

${ }^{5}$ Girolamo Tiraboschi, en la introducción al tomo I de su Historia, tiene que definir los límites geográficos del territorio cultural objeto de sus estudios: «Quando io dico di volere scriver la Storia della Letteratura Italiana, parmi ch'io spieghi abbastanza, di qual tratto di paese io intenda di ragionare» (Storia della letteratura italiana (2. edizione modenese riveduta ed accresciuta dall'Autore), I, Società tipográfica, Módena, 1786, pág. VI). La historiografía literaria se ha enfrentado desde sus comienzos a cuestiones sí lingüísticas, pero también geográficas y políticas, debido a la peculiar conformación e historia de la nación italiana. Sobre este tema véase el imprescindible ensayo de C. Dionisotti, Geografia e storia della letteratura italiana, Einaudi, Turín, 1967. 
La cuestión literaria y la política quedaron imbricadas desde los albores de la literatura vulgar, y en estrecha relación con otra cuestión, la lingüística, solo resuelta en el siglo Xx. Como es sabido, las discusiones acerca de la elección de una lengua común para la población peninsular, la «Questione della lingua», es un debate que arranca de Dante y toma carácter estrictamente político en el siglo XIX con Ascoli, Leopardi y Manzoni entre otros. La italiana es, pues, una «nación cultural» con una autoconsciencia que precede la formación política de la misma, un Estado endeble pero con una fuerte identidad basada en una tradición compartida, primero percibida por aisladas figuras intelectuales y artísticas para luego concretarse política y socialmente. Si por nación cultural cabe entender la interpretación que la crítica de Herder confirió al concepto ${ }^{6}$, el idioma es la impronta del espíritu y carácter de un grupo nacional, el Volksgeist (o, según la definición de Chabod, de un «alma nacional»), términos que cifran la gran novedad de la idea de nación entre finales del XVIII al XIX?.

En el ámbito editorial, durante el período resurgimental aparecieron proyectos que anticipaban la constitución de las Ediciones Nacionales, las cuales arrancarían oficialmente con la publicación de la obra completa de Galileo Galilei en 1887. En la primera década del siglo XIX se publican las Edizione delle Opere classiche italiane dedicadas a Francesco Melzi d'Eril, mientras que en 1843 se funda la importante colección «Biblioteca Nacional» del editor Le Monnier (Florencia), en la cual el espíritu del Risorgimento es ya patente. Son proyectos que apelaban a la idea romántica de nación, concepto precedente del de estado: la nacionalidad, para estos pensadores y mecenas culturales, se conformaba como un modo de ser de la vida comunitaria en su progreso histórico, una realidad de la contingencia histórica, lo que Georg Jellinek, jurista austriaco, teorizaría después como «derecho público subjetivo», por el que Nación es algo esencialmente subjetivo, un índice de un determinado estado de conciencia que aglutina a un grupo social en virtud de una cantidad de elementos de civilización comunes, entre los que destaca, sobre todo para el caso italiano, la literatura ${ }^{8}$, que, en cuanto cristalización de una tradición cultural compartida, ha participado desde siempre en la construcción de la unidad política. La importancia de la tradición queda reflejada en la misma conceptualización y periodización historiográfica italiana: se habla pues de Renacimiento, Resurgimiento para referirse a determinados periodos históricos, políticos y artísticos, teniendo pues la mirada puesta siempre en un pasado (latino, clásico) que debería ser el referente de un determinado espíritu nacional (la Storia della letteratura italiana de Tiraboschi arranca desde los Etruscos). La operación de las Ediciones Nacionales, caso ejemplar y casi único en Europa, está dirigida, pues, a la revalorización e institucionalización de esta tradición,

\footnotetext{
${ }^{6}$ Véase H. Kohn, Historia del nacionalismo, FCE, México, 1984, págs. 358-359.

${ }^{7}$ F. Chabod, L'idea di nazione, Laterza, Roma-Bari 1998, págs. 23 y sigs.

8 Véase Enciclopedia del diritto, Giuffrè, Milán, 1977, XXVII, pág. 803.
} 
a través de la edición oficial, crítica, última y definitiva de textos que abrazan la cultura italiana en su totalidad, sea científica, artística, filosófica. Y de seguir la historia de la publicación de las Ediciones Nacionales, analizando los criterios de edición adoptados o los nombres elegidos, se puede asimismo ver, en filigrana, la evolución de la idea de literatura nacional y, por ende, del concepto mismo de nación. Es significativo el hecho de que en los últimos años del XIX y en la primera década del Xx se privilegia a los autores del Risorgimento, así Cavour o Mazzini; con la llegada del nacionalismo fascista, se publicarán la opera omnia de Gabriele D'Annunzio, único caso de edición nacional en vida del autor, en la que se resalta su «fierissima italianità», o la de Giosuè Carducci; $y$, en la segunda posguerra, se asistirá a una progresiva descentralización política, que se traduce también, en el ámbito editorial, como aprobación de proyectos originales tal la publicación de la obra dialectal de Giuseppe Gioachino Belli (Poesie romanesche) o la opera omnia, también dialectal, de Salvatore Di Giacomo.

$\mathrm{Si}$, considerando la constitución de las varias Ediciones Nacionales, cabe ver en ellas cambiantes proyectos de política cultural, en la evolución de las mismas se puede advertir también el progreso de una nueva disciplina, la de la Filología italiana. No es casual que muchas de las ediciones nacionales se basen en la «filología de las variantes», concreción disciplinaria ecdótica típicamente italiana: la edición de Audisio de la Estética croceana es un ejemplo paradigmático. Esta «crítica nueva», como la definió Michele Barbi, y que tiene en Gianfranco Contini uno de sus fundadores (Come lavorava l'Ariosto, 1937), es una metodología que se adapta a los textos modernos y se centra en el proceso evolutivo de creación de la obra, testimoniando todas las fases de escritura que la colación de los testimonios permita. Se crea así una disciplina ad hoc para los textos modernos en lengua vulgar. La Filología italiana se colocaría al lado de las de más antigua tradición, como la clásica, la románica, la medieval, la humanística, la bíblica. Las Ediciones Nacionales son, pues, monumentos de este sector disciplinario. Confeccionando las Ediciones Nacionales en tanto que ediciones críticas, se confiere importancia al texto, equiparando los esfuerzos de edición de textos modernos a los de los clásicos con resultados de legitimación.

El concepto de «clásico» es relacionable con el de Edición Nacional. Un permanente debate acerca de la cualidad de clásico es el que oscila entre la referencia interna a la obra y la referencia a su institucionalización. La dialéctica entre ideología y estética a propósito del canon es reconducible al discurso de las Ediciones Nacionales, a sus criterios de selección-exclusión que, en el caso italiano, se fundan en el supuesto «valor de civilización» de una obra. Por ello, es condicio sine qua non para poder utilizar fondos públicos, la institución de unos comitati que evalúan la importancia histórica y cultural del autor seleccionado y, si procede, la institución de una comisión académica y científica que se encargaría de la constitución de un equipo de expertos en la materia. No será necesario entrar en comentarios acerca de estos procedimientos, tan 
habituales. Sea como fuere, y según dice Scotti, la historia de las Ediciones Nacionales está condicionada inevitablemente por «la storia dei rapporti fra Stato e cultura, in un settore apparentemente al di fuori di un piano di politica culturale ma dalla politica variamente condizionato»».

Eliot distinguía entre un clásico universal y otro tipo de clásico que lo es sólo en relación al resto de la literatura en su idioma: «dentro de sus limitaciones formales, el clásico debe expresar la mayor parte posible de la gama de sentimientos que representa el carácter del pueblo que habla esa lengua $\gg^{10}$. Quizás sea esta la definición de clásico que mejor se adapte a la operación de editar a un grupo de autores muy heterogéneos entre sí, pero que comparten un mismo idioma de expresión y, en cierta medida, una misma cultura. Las Ediciones Nacionales se fundamentan, en definitiva, sobre una idea de historia literaria nacional entendida en tanto que biblioteca.

\subsection{El caso español}

Si el caso español presenta una estructura académica estable, sin embargo no dispone de un concepto de Edición Nacional como el italiano. Una excepción, de gran relieve, fue la publicación de la opera omnia de Menéndez Pelayo por el Instituto de España en Edición Nacional: así la define el entonces ministro de Educación Nacional José Ibáñez Martín en el Prólogo a la Historia de las ideas estéticas en España, título que abre la serie en $1940^{11}$. El planteamiento de esta Edición Nacional es prácticamente el mismo que el de las italianas: el Consejo Superior de Investigaciones Científicas designó un equipo de expertos para coordinar la publicación de las obras completas (un total de 67 volúmenes en el arco de tiempo 1940-1974), presidido por Don Miguel Artigas, director de la Biblioteca Nacional y Don Enrique Sánchez Reyes, director de la Biblioteca de Menéndez Pelayo en Santander. Recuerda Pedro Sainz Rodríguez, ministro de Instrucción Pública en 1938: "siguiendo este criterio, decreté la publicación de las Obras de Menéndez Pelayo por el Instituto de España en Edición Nacional. Creo que, por un lado, hice un bien a su memoria y a la cultura española, pero, por otro, le rendí un mal servicio: muchos le combatieron sin conocerle, porque le consideraron banderín político. Al separarme radicalmente de la política del Alzamiento y del Gobierno dictatorial, en varias ocasiones he reivindicado la figura del gran polígrafo contra esa postura exagerada y tendenciosa que se le dio de un lado, provocando una reacción injusta en el otro» ${ }^{12}$. Y el hecho es que tras la publicación de la opera omnia de Pelayo

\footnotetext{
${ }^{9}$ M. Scotti y F. Cristiano, op. cit., pág. 263.

${ }^{10}$ T. S. Eliot, Sobre poesía y poetas, Icaria, Barcelona, 1992, pág. 70.

${ }^{11}$ En M. Menéndez y Pelayo, Historia de las ideas estéticas en España, I, CSIC, Madrid, 1940, págs. IX-XX.

12 «Advertencia preliminar», al Epistolario de Marcelino Menéndez Pelayo, 1, Fundación Universitaria Española, Madrid, 1982, pág. VIII.
} 
no se volvió a publicar ninguna otra Edición Nacional tout court. Sin embargo, se fundó una editorial, la Editora Nacional (el primer director fue Pedro Laín Entralgo), que, entre otras colecciones, se encargó de la publicación de textos pertenecientes a la tradición literaria y filosófica española. La Editora Nacional, de propiedad estatal, conoció en los años del tardofranquismo y de la transición una profunda transformación, lo cual auguraba la consolidación de una editorial pública en la España democrática que permitiese cuidadas y valiosas colecciones de clásicos españoles y universales y proyectos editoriales de dudosa rentabilidad (como la célebre colección Biblioteca de la Literatura y el Pensamiento Hispánicos), pero las presiones de grupos privados políticamente vinculados determinaron su liquidación tras la victoria electoral del PSOE en 1982. El cierre de Editora Nacional por el gobierno socialista implicó también la eliminación física de miles y miles de ejemplares y el subsiguiente beneficio de grupos y empresas emergentes o establecidos.

Anteriores proyectos de creación de una biblioteca de autores nacionales fue la benemérita Biblioteca de Autores Españoles, que concluye en 1872, subvencionada activamente por el Gobierno, así como Clásicos Castellanos, de 1910, que nació al amparo de la Junta de Ampliación de Estudios, organismo estatal. Quedó nonata la Biblioteca de Escritores Clásicos, un desiderátum del entonces presidente del Gobierno de la República Manuel Azaña. En el decreto publicado en la Gaceta el 23 de abril de 1936 se lee, con varios conceptos semejantes a los utilizados por los italianos:

[...] el Gobierno de la República, velando por la conservación y difusión de los monumentos de la lengua y literatura nacionales, en los que se reconocen los más gustosos frutos del espíritu español, y algunos de sus más preciados títulos en la historia de la civilización, ha acordado crear una biblioteca de escritores clásicos, dirigida, no solamente a poner buenos textos al alcance de un público formado de gentes letradas, sino a divulgación entre la juventud escolar y el pueblo. La necesidad de emprender esta obra de carácter nacional es evidente, si se considera el estrago que causa la corrupción del habla, y el provecho de acercar al conocimiento común las fuentes puras de la tradición literaria. Cree el Gobierno que el Estado puede y debe asumir la realización de esa obra.

Actualmente existe un proyecto de revalorización y fijación de la literatura en tanto que patrimonio nacional en la denominada Biblioteca Clásica de la $R A E$, que reunirá en ciento once volúmenes el núcleo esencial de la tradición literaria española e hispanoamericana hasta finales del siglo XIX, textos presentados en edición crítica o similar ${ }^{13}$.

${ }^{13}$ Sin embargo, en estas últimas décadas, verdaderos proyectos de ambición clásica pero novedosa hispánica y española son los que pueden verse en el ámbito privado, mediante dos ejemplos diversos y casi opuestos, gracias a las colecciones de Verbum Mayor y Biblioteca Castro, propiamente reconstructiva e investigadora la primera y elegante y limitadamente formal la segunda. 


\section{Croce en España: las traducciones}

Convendrá comenzar por recordar las versiones de la Estética de Croce en España ${ }^{14}$. La primera edición española data de 1912, a cargo de Francisco Beltrán. Es la traducción inicial de José Sánchez Rojas, quien se basó en la tercera edición italiana. Sin embargo, cuando el libro estaba a punto de imprimirse, Croce había iniciado ya la revisión de la cuarta, de lo cual tuvo noticia el traductor, que dejó una nota Post-scriptum en la que da fe de ello. Sánchez Rojas, periodista, escritor y traductor del italiano (hizo estancia doctoral en Bolonia) mantuvo relación de amistad con Miguel de Unamuno, que prologaría la primera edición española de la Estética. Ha de suponerse que fue el mismo Unamuno quien encargó o cuando menos propuso a Sánchez Rojas la traducción de la obra (Unamuno encargó otras traducciones del italiano a Sánchez Rojas, entre las que destacan las de Giovanni Papini; también tradujo el Príncipe de Machiavelli, La política clerical y la democracia de Romolo Murri y los ensayos La vida es sueño, Don Quijote y otros ensayos de Giosué Carducci). De Benedetto Croce, Sánchez Rojas tradujo el Breviario de Estética, que, a diferencia de la Estética, tuvo muchas ediciones en Espasa-Calpe (nueve en total, la última de 1985) y una reedición en la colección Planeta-Agostini, en 1992; también hizo versión de otra obra de Croce, España en la vida italiana del Renacimiento, publicada en Madrid, $1920^{15}$.

En el prólogo de Unamuno, de 1911, se lee:

[...] porque la Estética de Croce, no a pesar de ser una obra de robusta y segura filosofía, sino precisamente por serlo, es una obra fuertemente liberadora y sugestiva para un artista, una obra revolucionaria, y son los artistas y poetas los que ante todo deben leerla y meditarla ${ }^{16}$.

Es valoración muy afín a la perspectiva crítica croceana. Unamuno define la estética del pensador italiano en tanto que «obra de ciencia y obra de arte» y alaba la «simplicidad y coherencia casi matemática de su sistema». Indica también, sin embargo, varias objeciones, contraponiendo la racionalidad exquisitamente italiana de Croce (su «italianidad») al sentir místico del pensamiento español, cosa que le conduce a disentir sobre el lugar de la religión en el sistema filosófico del italiano. Pese a estas divergencias, cabe decir que Unamuno fue uno de los pensadores españoles que importó, directa o indirectamente, las ideas croceanas a España, aun para refutarlas o criticarlas. Así puede afirmar

${ }^{14}$ Sobre la relación Croce-España, véase el reciente estudio dirigido por Giuseppe Galasso, Croce e la Spagna, Editoriale Scientifica, Nápoles, 2011.

${ }_{15}$ Referencias bibliográficas de las traducciones de José Sánchez Rojas a obras de Benedetto Croce: España en la vida italiana del Renacimiento, Mundo Latino, Madrid, 1920; Breviario de Estética, Espasa-Calpe, 1938; y Breviario de estética: cuatro lecciones seguidas de dos ensayos y un apéndice, Planeta-Agostini, Barcelona, 1992.

${ }^{16} \mathrm{~B}$. Croce, Estética como ciencia de la expresión y lingüística general, ed. de P. Aullón de Haro y J. García Gabaldón sobre la versión de Á. Vegue i Goldoni, Ágora, Málaga, pág. 6. 
Eugenio D’Ors, en un artículo de 1951 aparecido en el periódico Arriba: «cronológicamente unidos, semejante en más de un aspecto de paralela acción, ha sido, en Italia, el maestro de Nápoles, Benedetto Croce lo que, entre nosotros, el maestro de Salamanca» ${ }^{17}$; aunque después señala fuertes diferencias entre ambos. Si Unamuno reservó casi siempre palabras de aprecio intelectual hacia el pensador napolitano, no se puede decir que la admiración fuese permanentemente recíproca. En efecto, Croce escribe sobre el libro Vida de don Quijote y Sancho según Miguel de Cervantes que «non si possono sopportare libri di [questa] sorta» ${ }^{18}$.

La segunda edición de la Estética croceana en España es de 1926. La publicó el editor Francisco Beltrán en su Librería Española y Extranjera, para la colección «Biblioteca moderna de Filosofía y Ciencias Sociales». Es la traducción de Ángel Vegue y Goldoni, que se basa en la quinta edición del texto croceano, que prácticamente puede darse como el texto italiano definitivo. Vegue y Goldoni, toledano, fue profesor de Historia del arte y vicepresidente de la sección de Bellas Artes (artes plásticas) del Ateneo de Madrid. Publicó algunos estudios sobre arte y literatura, entre los que cabe recordar Temas de arte y de literatura (Madrid, 1928) o la publicación de su tesis doctoral: Los sonetos "Al itálico modo» de Don Íñigo López de Mendoza: estudio crítico y nueva edición de los mismos (A. Marco, Madrid, 1911). También tradujo del francés El arte en España y Portugal del arqueólogo transalpino Marcel Dieulafoy (en la colección Historia general del Arte), publicado en Madrid, en 1920.

Hasta 1962 no habrá más ediciones de la estética croceana. Ese mismo año aparece en la Editorial Nueva Visión, de Buenos Aires, en la colección Arte y Estética dirigida por Alfredo Hlito y Francisco Bullrich ${ }^{19}$, una nueva edición parcial de la obra, de la parte teórica. La traducción utilizada es la de Ángel

${ }^{17}$ En V. González Martín, La cultura italiana en Miguel de Unamuno, Universidad de Salamanca, 1978, págs. 278-279.

${ }_{18}$ B. Croce, Letture di poeti e riflessioni sulla teoría e la critica della poesía, Laterza, Bari, 1950, pág. 53. Sobre la relación Croce-Unamuno, véase también Armando Savignano, «Il rapporto tra Croce e Unamuno», en Croce filosofo, II, a cura di G. Cacciatore, G. Cotroneo y R. Viti Cavaliere, Catanzaro, Rubbettino, 2003; C. Luigi Ferraro, «El concepto de la historia en Unamuno y Croce», en Miguel de Unamuno. Estudios sobre su obra, II (ed. de A. Chaguaceda Toledano), Ediciones Universidad de Salamanca, 2005, págs. 131-145; V. de Tomasso, «Unamuno e Croce: affinità e divergenze», Rivista di Studi Crociani, VIII, 1971, págs. 184-192.

${ }^{19}$ Alfredo Hlito, pintor y teórico del arte argentino, fundó en 1951, con Tomás Maldonado, la revista de arte militante (neovanguardista) Nueva Visión, vinculada a la editorial Nueva Visión en la que se publica el libro de Croce. Francisco Bullrich, miembro del comité editorial de la revista, es un arquitecto argentino. En la misma colección Arte y Estética se publican autores como E. Grassi, Arte y mito (1968), M. Bense, Estética: consideraciones metafísicas sobre lo bello, 1973; B. Bosanquet, Historia de la estética, 1970; W. Worringer, El arte y sus interrogantes, 1959; R. Hans, Historia del dadaísmo, 1973; W. Hess, Documentos para la comprensión del arte moderno, 1967; J. J. Winckelmann, Lo bello en el arte, 1958; L. Mumford, 
Vegue y Goldoni de 1926, si bien actualizada por León Dujovne ${ }^{20}$ (la traducción del prólogo de Adelchi Attisani es de Manuel Belloni). La revisión de la versión de Vegue se hizo sobre la 10a. edición italiana (Laterza): en efecto el editor añade una nota preliminar de Croce (enero de 1941) que en la precedente versión no estaba, por obvias razones cronológicas. En cuanto al texto, las diferencias son mínimas (modernización de la puntuación, corrección de algún que otro giro lingüístico, normalización de las cursivas, ligeros cambios en los conectores textuales, etc.). Las modificaciones léxicas son muy escasas y no afectan a las palabras clave del pensamiento croceano. A modo de ejemplo, valga la sustitución de «inteligencia» (versión de Vegue) por «intelecto».

No se incluye el prólogo de Unamuno, que viene sustituido por una introducción del filósofo y pedagogo italiano Adelchi Attisani, autor de varios trabajos de materia croceana ${ }^{21}$. Es una introducción bastante didáctica, en la que se ilustran los principales argumentos y tesis de Croce, siguiendo el orden con el que el filósofo italiano los argumentó en su texto. También trata de la fortuna editorial de la obra y del desarrollo del pensamiento de Croce tras la publicación de su Estética, aludiendo, pues, a la estructuración de la Filosofía del Espíritu en tanto que sistema filosófico. Por lo demás, en 1982 aparece en la Universidad Autónoma di Sinaloa, Culiacán (México), una reproducción mecánica y sin referencia legal de la versión de 1926, que no presenta mayor novedad.

Necesaria y con una extensa introducción actualizada es la edición de Aullón de Haro y García Gabaldón, quienes reproducen el texto de la traducción de Vegue y Goldoni, modernizado y poco más, pues los editores consideraron muy buena la versión del 26 (se han corregido italianismos léxicos y sintácticos, una cincuentena de erratas, y se ha modernizado la acentuación ortográfica). El texto se publica en la editorial Ágora, en la colección Hybris, que incluye en su catálogo textos diversos de estética pura, teoría del arte, filosofía.

La introducción, redactada por Aullón de Haro y Gabaldón, se estructura según la explicación, a partir del texto y complementada por el estudio de los

Arte y técnica; G. Dorfles, Constantes técnicas de las artes, 1958; L. Venturi, Cuatro pasos hacia el arte moderno: Giorgione, Caravaggio, Maner, Cézanne, 1960.

Para la relación Croce-Argentina, fundamental fue la labor docente e investigadora de Coriolano Alberini (1886-1960), profesor de Gnoseología y Metafísica en la Universidad Nacional de La Plata. Introductor de Bergson y Croce en la Argentina, fue secretario técnico del Primer Congreso Nacional de Filosofía de Mendoza, de 1949. Véase C. Beorlegui, Historia del pensamiento filosófico latinoamericano, Deusto, Bilbao, ${ }^{32010}$, págs. 444-450. Otra personalidad intelectual importante para la importación de las ideas estéticas croceanas es el filósofo Alejandro Korn.

${ }^{20}$ León Dujovne, escritor y filósofo argentino, publicó en 1968 un estudio sobre la filosofía de la historia de Croce: El pensamiento histórico de Benedetto Croce, Rueda Editor, Buenos Aires, 1968.

${ }_{21}$ A. Attisani, Interpretazioni crociane: arte, totalità, moralità, Messina, La Sicilia, 1932; Espressione ed esperienza: premesse ad una didattica dell'attività espressiva, Urbino, 1959; Introduzione all'Estetica di Benedetto Croce, Bologna, Cappelli, 1961; Sull'Estetica di B. Croce: due note, Catania, Studio Editoriale Moderno, 1924; «Pagine di pedagogia di B. Croce», Rassegna di pedagogia, 1-2, enero-abril, 1950. 
escritos estéticos sucesivos, de los conceptos clave de la estética de Croce, a saber, «Intuizione ed espressione», «Espressione e comunicazione», «Retorica, Grammatica e Filosofia del linguaggio» (así se subdivide el capítulo II, «El pensamiento estético de Croce»). Conscientes de que «el lugar histórico y cultural croceano se encontraba y, tal vez, se sigue encontrando muy enmarañado» ${ }^{22}$, los editores piensan en la edición del texto como esencial a fin de resituar la aportación teórica del idealismo estético y poético y proponer una filología filosófica, pasando por una reformulación comparatista y universalista de las ciencias humanas, algo que ha de empezar a partir de la revalorización de personajes intelectuales como Juan Andrés, Jean Paul Richter, Karl Krause, Friedrich Shiller, etc. De otra parte, la edición de Croce entra en esa operación de revitalización directa, a través de los textos, de la gran escuela filosófica y literaria hispano-italiana (Luzán-Muratori, la Escuela Universalista compuesta por Andrés, Eximeno y Hervas, Croce hispanista, etc.), una alternativa viable y efectiva al monopolio anglosajón sobre la crítica y la teoría literaria, dominio que en ocasiones implicó una depauperación de la disciplina.

Pero también la edición de la Estética croceana se conforma como un modo «de la estética idealista, de su integridad y acabamiento disciplinario frente al esteticismo decadente, y un sentido de intemporalidad o salvaguarda de la materia que Adorno quedará impelido a trasladar a otros cursos posteriores de la historia y las artes». En definitiva, «Croce representa otra vez, aun con los distingos necesarios, un singular centramiento idealista del espíritu del arte»²3.

Además de al español, la Estética de Croce se ha traducido a muy diversos idiomas: albanés, checo, chino (tres diferentes versiones), estonio, francés, japonés, griego, inglés, noruego, rumano, ruso, serbo croata, alemán, turco, húngaro. El caso más llamativo quizás sea el inglés, donde encontramos varias ediciones: si la primera es de 1909, a cargo del traductor Douglas Ainslie (la versión más difundida), en las décadas de los 50 y 60 se subsiguen muchas ediciones, sobre todo en Nueva York. La difusión de las ideas estéticas croceanas se debe sobre todo a Collingwood, para quien el concepto estético de «expresión» se asume esencial en Los principios del arte. Para analizar la recepción de Croce en Estados Unidos resulta decisiva la reseña que efectúa Moss, Benedetto Croce negli Stati Uniti (en I progressi della filosofia nell'Italia del Novecento, Morano, Nápoles, 1992). La autora considera emblemático el caso del libro Arte como experiencia de Dewey, que contiene claros ecos de la estética croceana, si bien el autor no lo declaró nunca. En los años 20 y 30 predominan las ideas historiográficas croceanas: Carl Becker y Charles Beard son los intelectuales que más difundieron y desarrollaron estas ideas. Importante e influyente se revela también la atención prestada por Hayden White a la teoría historiográfica del filósofo partenopeo.

\footnotetext{
${ }^{22}$ B. Croce, op. cit., pág. IX.

${ }^{23}$ Loc. cit., pág. XI.
} 
El caso de Francia es significativo: si bien la francesa fue una de las primeras traducciones a un idioma extranjero (1904, traducida sobre la 2a. edición italiana por Henry Bigot), no se volvió a traducir ni imprimir el texto, quizás debido a la influencia y difusión de las ideas formalistas y estructuralistas en el ambiente académico transalpino ${ }^{24}$. Sin embargo, hay que mencionar el libro Essais d'esthétique: textes choisis de Benedetto Croce, traducidos y presentados por Gilles A. Tiberghien, publicado en Gallimard en 1991; y una edición reciente del Bréviaire d'esthétique vertido por Georges Bourgin y prologado por Gilles A. Tiberghien, de 2005. En Francia, como en España, las ideas de Croce se difundieron más a través de su Breviario (cuyas ediciones españolas son 10 en total), que gracias al libro principal.

\section{Sobre la edición nacional de Benedetto Croce}

La edición nacional de la opera omnia de Benedetto Croce se instituye el 14 de agosto de 1981, con la creación de un comité científico presidido por Mario Scotti25, conocido italianista y croceano ${ }^{26}$, editor de Ugo Foscolo o Silvio Pellico ${ }^{27}$. A partir de 2008, tras el fallecimiento de Scotti, le sucede Gennaro Sasso, que sobre Croce publicó, entre muchos otros ensayos y artículos, Benedetto Croce interprete di Macchiavelli (Malfasi, Milán, 1953) y Benedetto Croce: la ricerca della dialettica (Morano, Nápoles, 1975).

El plan editorial de la edición de la opera omnia de Croce consta de dos secciones iniciales. La primera presenta las obras dispuestas por el mismo Croce («Corpus disegnato per l'autore»), es decir, el plan del autor para la publicación en los años 50 de su obra completa en editorial Laterza ${ }^{28}$, con la que colaboró activamente. El Corpus se divide en las secciones: «Filosofía dello spirito», «Saggi filosofici», «Scritti di storia letteraria e política», «Scritti vari». El objetivo de los editores fue seguir el plan laterziano, complementado con las adiciones póstumas que allí no pudieron entrar pero que respetarían el deseo

${ }^{24}$ Véase a propósito G. Pagliano Ungari, Ricerche sulla fortuna di Benedetto Croce in Francia, Didier, 1965; y S. Romano, Per la conoscenza di Croce in Francia, Istituto italiano di studi storici, Nápoles, 1984.

${ }^{25}$ Los restantes miembros son: Piero Craveri, secretario; Ettore Bonora, Enzo Bottasso, Alda Croce, Raffaello Franchini, Franco Gaeta, Aldo Garosci, Gaetano Mariani, Maurizio Mattioli, Giovanni Nencioni, Benedetto Nicolini, Alfredo Parente, Giorgio Petrocchi, Giovanni Pugliese Carratelli, Rosario Romeo, Gennaro Sasso, Vittorio Stella, Leo Valian.

${ }^{26}$ El acercamiento de Scotti a la obra de Croce fue sustancialmente inherente a la crítica literaria del filósofo italiano: recordamos el estudio Croce e Foscolo, de 1980, o L'Arcadia nella riflessione critica di Croce, Fubini e Binni, 1991.

${ }_{27}$ Para los criterios de edición adoptados, véase Edizione nazionale delle opere di Benedetto Croce, Bibliopolis, Nápoles, 2010.

${ }_{28}$ Véase F. Nicolini, L'Editio ne varietur delle opere di Benedetto Croce: saggio bibliografico con taluni riassunti o passi testuali e ventinove fuori testo, Arte tipográfica, Nápoles, 1960. 
del autor, así el epistolario Croce-Vossler, los Aneddoti, las Pagine sparse, las Nuove e las Terze pagine sparse, los Scritti e discorsi politici.

La segunda sección, «Scritti sparsamente editi», recoge ensayos, artículos, reseñas de revistas y periódicos; la obra I teatri di Napoli en la edición de 1891, la Bibliografía vichiana y Gli scritti di Francesco de Sanctis. Siguen otras cuatro partes: la tercera de traducciones de Hegel, Basile, Erasmo, etc.; la cuarta, dedicada a los Taccuini di lavoro; la quinta tenía que recoger el epistolario; la sexta la bibliografía de y sobre Croce y la séptima y última parte se compone del índice analítico-sistemático. Dados los retrasos en la publicación de la obra completa y la dificultad de plantear una edición crítica de tan gran mole de material, el comité presidido por Sasso renunció a la publicación del opus epistolarum y a la edición crítica de los Taccuini, cuya inestabilidad textual atascó los trabajos de edición.

Todos los tomos de la Edición Nacional recogen ediciones críticas de las obras de Croce ${ }^{29}$ : el principal objetivo del comité científico, como escribe Scotti, era ofrecer los resultados de la revisión textual en sus diferentes fases de redacción. El aparato tiene que ser no un aval razonado del texto, sino el espejo de un proceso diacrónico. En la edición crítica se consideran sólo los impresos, no manuscritos, es decir solo variantes de las impresiones: irónicamente, los textos de Croce se publican con el método filológico de la crítica de las variantes, metodología por la que el napolitano tuvo consideración no muy entusiasta ${ }^{30}$.

La impostación filológica conferida a esta edición permite sopesar el rigor estilístico al que Croce sometía sus textos, dispuesto a cambiar una oración aun sólo por mejorar su eufonía. Y ello es significativo para la Estética, que Croce no dejó de modificar y corregir durante toda su vida. Giovanni Spadolini afirmó que Croce:

[...] ha raccolto tutti i suoi scritti con metodo certosino, con pazienza esemplare [...]; ha preventivato tutto; ha calcolato tutto. Quasi nel timore che mani esterne si sovrapponessero alla sua inimitabile costruzione intellettuale ${ }^{31}$.

Croce publicaría en 1918 la primera edición del Contributo alla critica di me stesso, la «storia della [sua] vocazione o missione» ${ }^{32}$ y a partir de 1906 comenzaría a recopilar anotaciones diarias reunidas luego en los Taccuini di lavoro.

${ }^{29}$ El epistolario crociano va publicándose gracias a la labor de la Fondazione Biblioteca Benedetto Croce, el Instituto Italiano per gli Studi Storici y la editorial Il Mulino.

${ }^{30} \mathrm{~B}$. Croce, «Illusione sulla genesi delle opere d'arte documentabili dagli scartafacci degli scrittori», Quaderni della critica, III, noviembre 1947, págs. 93-94.

${ }^{31}$ G. Spadolini, Per l'edizione nazionale delle opere di Benedetto Croce, Istituto Italiano per gli Studi Storici, Nápoles, 1991, pág. 10.

${ }^{32}$ B. Croce, Contributo alla critica di me stesso, Ricciardi, Nápoles, 1918, pág. 4. 
Croce pertenece, pues, a esos autores, sistemáticos o no, que tienen muy claro desde el comienzo la unidad y la coherencia que posee el conjunto de sus obras; autores obsesionados en la construcción de su personalidad intelectual. El suyo es un caso parecido al de Menéndez Pelayo o Alfonso Reyes, grandes polígrafos que plantearon en vida la organización de su obra. En estos casos, como en otros de autores de ficción (el ejemplo de Balzac es paradigmático), la opera omnia se configura como una especie de género literario en constelación, un ideal heurístico de búsqueda experimental, un ideal y una utopía.

\subsection{La edición nacional de la Estética}

La Edición Nacional de la Estética de Croce se divide en tres tomos: el segundo reproduce la 2. edición, impresa en Palermo, editorial Sandron; el primero recoge la última edición, la 9a, en vida del autor (1950), impresa por Laterza; el último reúne el estudio de la editora y el aparato crítico que da razón de ser de esta edición crítica. Así, la «Nota al testo» incluye el epígrafe «Genesi e storia del testo», dedicado a la génesis y a los estudios preparatorios que llevarán a Croce a redactar su obra maestra; un capítulo sobre las vicisitudes editoriales («Le vicende editoriali») con exhaustivo estudio de la novena edición. Además, incorpora otras tres secciones: dedicadas a las traducciones, a varios testimonios, tanto impresos como manuscritos, y a criterios de edición, donde Audisio expone su modus operandi filológico. El «Aparato crítico» ocupa más de 300 páginas. Siguen unos apéndices, en los que se presentan el cotejo, por ejemplo, de los «sommarietti» de las ediciones 1904 y 1950 o el «Indice delle cose» (Índice temático) de la edición de 1904.

Interesante es la última sección, «Indici». Además de un índice onomástico que se refiere a las dos ediciones propuestas, se ofrece un «Indice dei riferimenti, dei rinvii e delle citazioni», que completa y detalla los extremos bibliográficos que en las notas a pie de página y en el Apéndice de la obra aparecen abreviados. Audisio, en el límite de lo posible, ha utilizado los libros manejados por el mismo Croce, conservados en la fundación Biblioteca Benedetto Croce. Podemos así ver, compendiado y organizado, todo el background bibliográfico que permitió la composición de la principal obra estética croceana. En cuanto a la bibliografía española, el esteta italiano consultó obras de Esteban de Arteaga (Investigaciones filosóficas sobre la Belleza ideal, considerada como objeto de todas las artes de imitación), Miguel de Cervantes (Don Quijote), Feijóo y Montenegro (Theatro critico universal), Baltasar Gracián (Arte de ingenio, tratado de agudeza; El héroe, el discreto; Obras), Francisco Sánchez de las Brozas (Minerva seu De causis linguae Latinae commentarius, 1587), George Santayana (un artículo que escribió en 1903 sobre la Estética croceana, publicado en el Journal of comparative literature), Iñigo López de Mendoza Marqués de Santillana (Obras), Juan de Valdés (Diálogo de las lenguas), Lope de Vega (Rimas), Juan Luis Vives (De causis corruptarum 
artium; De ratione dicendi libri tres) y Menéndez y Pelayo, Historia de las ideas estéticas en España. Croce define la Historia de las ideas estéticas en España en tanto que «opera capitale per ciò che concerne gli scrittori spagnuoli» y que presenta, en la parte general, «buone trattazioni di argomenti tralasciati di solito nelle altre storie» ${ }^{33}$. Croce se vale del libro de Menéndez Pelayo también para compilar el capítulo XIII, dedicado a los estetas alemanes menores, y para el capítulo XVI «La estética degli epigoni», dedicada a la última fase de la Estética metafísica. También lo cita en nota repetidamente en el cap. XIX, «Sguardo alla storia di alcune dottrine», sobre todo para las referencias españolas. En el cap. II, sobre la estética de San Agustín y de los primeros escritores cristianos, la referencia explícita, en el apéndice bibliográfico, es nuevamente hacia Menéndez Pelayo, así como para la tradición de las ideas platónicas y neoplatónicas en el Medievo y en el Renacimiento (según el juicio de Croce, Menéndez Pelayo trata este argumento «più ampiamente e meglio di tutti»). Acerca del cap. XIV dedicado a la historia de la Estética francesa en el siglo XIX, Croce sostiene que «la migliore esposizione» es la del polígrafo cántabro.

Como hemos repetido, el estudio de Audisio es estrictamente textual, pero, como se ha podido comprobar, el aparato crítico no se limita a ser mero instrumento de organización filológica, pues arroja luz sobre el proceso genético de la obra y, en nuestro caso, sobre las varias (re)escrituras que el autor hizo de su texto. En este sentido es de recordar la operación más significativa, es decir la preparación de la tercera edición (1908), primera en Laterza. Esta edición señala, además, el ingreso de la obra en el sistema de la Filosofía del Espíritu. Croce, en aquel entonces, atendía a la composición de la Filosofía de la práctica y a la reescritura de la Lógica. Un texto decisivo que se interpone entre la segunda y tercera edición es la memoria enviada para el Congreso de filosofía de Heidelberg en 1908, con el título L'intuizione pura e il carattere lirico dell'arte.

Como explicita Croce en la Advertencia de 1907, los capítulos que más padecen modificaciones y rectificaciones son el x y el XII. Allí se refleja la concepción del arte como «intuición pura» concretados en la memoria antes citada. Son elementos que indican este nuevo logro de la filosofía croceana: en tres ocasiones se añade el adjetivo «pura» al término «intuición», cosa relevante para definir el nuevo concepto de «poesía» como «intuición pura». En la memoria enviada al congreso de filosofía alemán se lee la siguiente difinición:

l'intuizione pura, non producendo concetti, non può, dunque, rappresentare se non la volontà nelle sue manifestazioni, ossia non può rappresentare altro che stati d'animo. E gli stati d'animo sono la passionalità, il sentimento, la personalità, che si trovano in ogni arte e ne determinano il carattere lirico ${ }^{34}$.

${ }_{33}$ B. Croce, Estetica come scienza dell'espressione e linguistica generale, II, Edizione 1950 (ed. de F. Audisio), Bibliopolis, Nápoles, pág. 565.

${ }_{34}$ B. Croce, «L'intuizione pura e il carattere lirico dell'arte», La Critica. Rivista di Letteratura, Storia e Filofosia, 6, 1908, pág. 335. 
La intuición pura es, sustancialmente, liricidad. En esos pequeños cambios léxicos se esconde, pues, la evolución y el progresivo afinamiento de la teoría croceana según quedan registrados en la gran edición crítica que comentamos.

La Estética de Croce, como viene a recordar Audisio en la conclusión de su estudio, es un auténtico clásico y muy influyente pero sometido a postergación como consecuencia de las posteriores imposiciones de la crítica gramsciano-marxista, fenomenológica, psicoanalítica y sociológica... Ahora bien, la supuesta «inactualidad» de las ideas estéticas croceanas permanece como alternativa, idealista de tradición románica, al decadentismo de la crítica y a la confusa teoría literaria y artística actual. 